\title{
La conversion prohibée : mariages mixtes et politiques de conversion dans le champ religieux juif argentin
}

\section{Sébastien Tank-Storper}

\author{
OpenEdition \\ Journals \\ Édition électronique \\ URL : http://journals.openedition.org/assr/3262 \\ DOI : 10.4000/assr.3262 \\ ISSN : $1777-5825$ \\ Éditeur \\ Éditions de l'EHESS
}

Édition imprimée

Date de publication : 1 décembre 2005

Pagination : 123-142

ISBN : 2-7132-2045-9

ISSN : 0335-5985

Référence électronique

Sébastien Tank-Storper, «La conversion prohibée : mariages mixtes et politiques de conversion dans le champ religieux juif argentin », Archives de sciences sociales des religions [En ligne], 137-132 I juillet décembre 2005, mis en ligne le 30 juin 2008, consulté le 01 mai 2019. URL : http:// journals.openedition.org/assr/3262; DOI : 10.4000/assr.3262

Ce document a été généré automatiquement le 1 mai 2019.

(ㄷ) Archives de sciences sociales des religions 


\title{
La conversion prohibée : mariages mixtes et politiques de conversion dans le champ religieux juif argentin
}

\author{
Sébastien Tank-Storper
}

Dans le foisonnement de responsa et décisions juridiques qui compose la littérature rabbinique, le décret de 1927 du rabbin argentin d'origine syrienne Shaul David Setton prohibant "pour toujours" toute conversion réalisée sur le sol de la République argentine revêt un caractère particulier. Rares sont les décisions juridiques qui figent un interdit sans condition ni limite temporelle, exceptée peut-être celle promulguée en 1830 par Moses Sofer (plus connu sous le nom de Hatam Sofer), au fondement de l'ultraorthodoxie moderne, visant à exclure toute innovation halakhique. Par-delà son originalité, le décret de prohibition argentin se révèle particulièrement intéressant si l'on s'en réfère au contexte de sa promulgation, aux logiques qui ont présidé à sa rédaction, à la manière dont il a été reçu et aux conséquences que son application a eues sur la structuration du judaïsme argentin. Censé au départ lutter contre les mariages mixtes et les « conversions sauvages », il n'eut aucune incidence réelle sur le taux d'exogamie de la judaïcité argentine (qui fut, dès les débuts, de l'ordre de 40 à $50 \%$ ). Par contre l'imposition progressive de la prohibition des conversions permit l'homogénéisation idéologique des communautés juives argentines, jusque-là relativement atomisées. Finalement, plus qu'un outil permettant de renforcer les frontières de la communauté juive dans un contexte de grande porosité avec le milieu non juif, le décret du rabbin Setton se révéla un formidable levier normatif au profit d'une orthodoxisation croissante du champ religieux juif argentin. À tel point que, aujourd'hui encore, malgré un nombre infiniment restreint de demandes de conversions adressées aux institutions argentines, le respect ou le non respect de ce décret focalise l'ensemble des débats entre les différentes tendances institutionnelles du judaïsme argentin, notamment entre orthodoxes et institutions de sensibilité libérales. Retracer la destinée de ce décret, c'est ainsi en partie 
retracer l'histoire de l'implantation juive en Argentine, et surtout de ses institutions religieuses.

L'immigration juive en Argentine : quelques repères historiques

2 La présence juive en Argentine est insignifiante jusqu'au milieu du XIX ${ }^{\mathrm{e}}$ siècle. Le pays est alors essentiellement peuplé de migrants espagnols, dont la population juive reste très marginale ${ }^{1} .1853$ marque un tournant dans l'histoire du pays, quand Buenos Aires rejoint la fédération argentine et qu'est initiée une politique active de peuplement. La devise nationale se résume alors à l'expression «Gobernar es poblar " [ gouverner, c'est peupler »], si bien que fut inscrite dans la constitution elle-même l'interdiction formelle de limiter l'immigration ${ }^{2}$. Quelques années plus tard, en 1876, la loi Avellaneda - ou loi sur l'immigration - régularise le processus d'absorption des immigrants. L'hôtel des immigrants, situé dans le port de Buenos Aires, a pour charge d'accueillir et de nourrir les nouveaux arrivés, ainsi que de leur fournir des billets de train pour se rendre vers leur destination finale dans l'intérieur du pays. De 1880 à 1930, un flux continu mène de nombreux candidats de l'Europe vers l'Argentine, avec un total estimé à plus de trois millions d'immigrants en 50 ans. Parmi eux, on compte une grande majorité d'Italiens et d'Espagnols, mais aussi des Français, des Russes, dont 5 \% de juifs.

Jusque dans les années 1890, les quelque 1500 juifs du pays, principalement regroupés dans la Capitale Fédérale ${ }^{3}$, étaient originaires de France et d'Allemagne et travaillaient essentiellement dans la banque et le secteur tertiaire naissant ${ }^{4}$. Ce sont eux qui, dès 1862 , fondèrent la première communauté juive du pays, à Buenos Aires, sur l'actuelle calle Lavalle.

4 La judaïcité argentine prend réellement de l'ampleur à partir de 1889, avec l'arrivée dans le port de Buenos Aires d'environ 1000 juifs venus principalement d'Europe de l'Est (Russie et Roumanie) et de Turquie. Dès lors, un flot continu vint gonfler la population juive du pays : elle était de 10000 en 1895, 100000 à la veille de la Première Guerre mondiale et plus de 200000 à la fin des années $1920^{5}$. Dans leur grande majorité (82,6 \%), ces immigrants venaient d'Europe centrale (Pologne, Russie, Roumanie, Lituanie, Lettonie, etc.). On recensait également une immigration séfarade et orientale (environ $10 \%$ ), originaire en premier lieu de Syrie, du Liban, de Turquie, du Maroc et d'Espagne. Sous l'impulsion du Baron Hirsch notamment, un certain nombre rejoignait les colonies agricoles de l'intérieur du pays et formait des villages presque exclusivement juifs, dont les plus connus restent Moisesville et Basavilbaso ${ }^{6}$. Il n'en demeure pas moins que les grandes villes attiraient la part la plus importante de ces migrants. Les conditions de vie dans la Pampa argentine étaient souvent difficiles et de nombreux colons quittaient les villages pour se fixer dans les centres urbains comme Rosario, Córdoba, Santa Fé et majoritairement - Buenos Aires.

5 Une dernière vague migratoire se produisit entre 1933 et 1948, conséquence directe des persécutions que subirent les juifs d'Europe durant cette période, et ce malgré la politique de fermeture adoptée par l'Argentine dès le début des années $1930^{7}$. Selon les sources, de 28000 à 38000 juifs, allemands pour la plupart ${ }^{8}$, entrèrent en Argentine entre 1933 et 1945 , soit environ $10 \%$ de la judaïcité argentine totale.

6 À partir de cette période, les lois restrictives adoptées par le gouvernement argentin en matière d'immigration freinèrent considérablement l'afflux de juifs dans le pays. La " communauté » argentine se stabilisa alors autour d'une fourchette allant, selon les estimations, de 215000 âmes pour la plus basse ${ }^{9}$ à environ 500000 pour la plus haute ${ }^{10}$, 
pour une population argentine totale de 36,7 millions en 2001 - soit une population juive qui représente entre 0,6 et $1,4 \%$ de la population argentine. Selon les cas, la diaspora juive argentine arrive donc de la dixième à la troisième place mondiale, derrière celle des États-Unis et de la France, et juste devant la Russie.

Un judaïsme « congrégationaliste » et anomique (1862-1930)

7 Les premiers temps de l'implantation juive en Argentine furent marqués par une forte tendance modernisatrice dont l'une des conséquences majeures fut le large désinvestissement religieux et le nombre très élevé de mariages mixtes, si bien que l'on a coutume de dire que les juifs laissaient leurs téfilin dans le bateau qui les menait dans le Nouveau Monde. À la fin du XIX siècle et au début du XXe, alors que la récente immigration juive n'était pas encore clairement organisée, régnait en matière de mariages mixtes et de conversion ce que les chroniqueurs ont par la suite appelé «la grande confusion ». Selon les différentes sources historiques, les premières heures du judaïsme argentin témoignent d'importants taux de mariages exogames, à l'exemple du premier rabbin de la Congregación Israelita de la República Argentina (CIRA, première institution juive d'Argentine, fondée en 1862) qui épousa une chrétienne et eut des enfants baptisés dans le catholicisme ${ }^{11}$. Il n'était d'ailleurs pas le seul. Victor A. Mirelman note que de nombreux dirigeants de la CIRA étaient mariés à des non-juives ${ }^{12}$. Il est également fait mention de conversions "sauvages» effectuées par n'importe quel membre des différentes synagogues, sans que les principes halakhiques soient respectés ni que la légitimité des rabbins dépasse le cadre de leur communauté. Entre 1906 et 1927, dans certaines synagogues, les convertis étaient acceptés sans autre formalité qu'une lettre attestant qu'ils s'étaient convertis de leur propre volonté et qu'ils promettaient d'éduquer leurs enfants dans la tradition juive ${ }^{13}$.

Ces taux d'exogamie importants et ces nombreuses conversions peuvent révéler la faiblesse de l'identité juive de ces migrants et, paradoxalement, sa vitalité. Essentiellement composée de jeunes hommes, la population juive arrivant en Argentine connaissait en effet un sexe-ratio très déséquilibré qui rendait nécessaire l'ouverture du marché matrimonial, et ce d'autant plus dans les petites communautés de l'intérieur ${ }^{14}$. Le mariage mixte pouvait donc être une nécessité plus qu'un choix, et la conversion signifier la volonté des conjoints juifs de ne pas s'éloigner du cadre communautaire en intégrant formellement les épouses non juives dans le giron du judaïsme, d'autant plus que l'existence d'un mariage civil ne donnait aucun caractère obligatoire à l'homogénéisation religieuse du couple.

En revanche, les modalités par lesquelles étaient réalisées ces conversions signifiaient pour leur part une certaine distance avec les principes de la tradition. Rappelons qu'en substance le Shoulkhan Aroukh stipule : $1^{\circ}$ - que le rituel de conversion (mikveh ${ }^{15}$ plus circoncision pour les hommes) est un acte irrévocable qui, s'il est mené dans les formes, ne peut être contesté ${ }^{16} ; 2^{\circ}$ - que le candidat doit être motivé par une intention pure (le shem shamaïm, défini en négativement comme ne pas vouloir se convertir pour obtenir un bénéfice ultérieur - argent, pouvoir, femme ou homme -); $3^{\circ}$ - que le ou la converti(e) s'engage à respecter les mitsvot (kabalat ol mitsvot) et $4^{\circ}$ - que l'acceptation d'un candidat sincère est une obligation. Il précise également que le rituel de conversion doit être mené par trois hommes connaissant parfaitement les textes codifiant le giyyur (processus par lequel un non juif est fait juif), à savoir, selon la coutume, par des rabbins communément reconnus pour leur compétence et leur savoir. 
10 Les pratiques de conversion en cours dans les premiers temps de l'implantation juive en Argentine, où le passage s'attestait le plus souvent par une simple auto-déclaration, s'éloignaient ainsi significativement des prescriptions des principaux textes codifiant la conversion. Ces écarts dépassaient d'ailleurs le seul cadre des conversions. Jusqu'au début des années 1920, la Jevra Kedusha ${ }^{17}$ acceptait dans son cimetière les conjoints non juifs des membres de l'association et de nombreux mariages mixtes étaient célébrés - après une conversion symbolique - dans les communautés séfarades ${ }^{18}$. Le judaïsme argentin prenait ainsi l'allure d'un judaïsme symbolique et conciliant, qui n'avait d'autre solution que de s'adapter aux contraintes de la modernité dans laquelle s'engouffrait la majorité des juifs argentins ${ }^{19}$.

11 Cette situation était en grande partie provoquée par le formidable morcellement des synagogues, typique des pays d'immigration qui accueillent des juifs d'origines diverses. En 1901, on comptait 23 lieux de culte israélite dans la seule ville de Buenos Aires pour environ 10000 âmes juives ${ }^{20}$. Les rabbins, pas ou peu formés, possédaient à quelques exceptions près une connaissance très relative de la loi et n'avaient pas d'autre choix que de proposer un judaïsme de type congrégationaliste où les règles religieuses étaient fixées plus par la communauté assemblée que par l'autorité d'un texte. Si, par de nombreux aspects, le judaïsme des premières années de l'implantation argentine pouvait ainsi sur de nombreux points s'approcher du judaïsme libéral, il s'en distinguait cependant en premier lieu par son absence totale de formulation idéologique. C'était, bien davantage, un judaïsme abandonné à lui-même qui tentait avec plus ou moins de réussite de faire perdurer quelques principes mal maîtrisés.

Le décret de prohibition des conversions du rabbin Shaul David Setton (1927)

En 1927, le rabbin d'origine syrienne Shaul David Setton en charge de la communauté Hesed Schel Emeth Sefaradit - communauté syrienne - de Buenos Aires (soutenu par le rabbin ashkénaze de Moisesville Aharon Halevi Goldman ${ }^{21}$ et avec l'approbation des grands rabbins séfarades et ashkénazes de Palestine - respectivement Jacob Meir et Abraham Isaac Kook - ainsi que du rabbin Judah Leib Zirelson de Kishinev, l'un des fondateurs de l'Agudat Israël), édicta la prohibition totale et définitive de toute conversion à l'intérieur des frontières de la République argentine. L'objectif explicite de ce décret était de mettre de l'ordre dans ces pratiques matrimoniales exogames ainsi que dans le traitement institutionnel des conversions, qui étaient perçues comme des dangers pour la pérennité de l'identité juive en Argentine :

La vie à Buenos Aires est particulièrement débauchée et chacun y fait ce qui lui plait ; il n'y a aucune autorité rabbinique écoutée et respectée, ni de rabbin nommé par le gouvernement ou par la communauté juive elle-même. En conséquence, quiconque le désire prend pour épouse une non juive sans qu'elle se convertisse, ou choisit des individus au hasard pour servir de témoins lors de la «conversion ». Et ils ont des enfants qui ne réunissent pas les conditions pour être juifs, bien que leurs parents naturels prétendent qu'ils ont été convertis. Si quelqu'un leur demande en présence de qui eut lieu la dite conversion, ils répondent effrontément: «qui l'a nommé rabbin [celui qui pose la première question] »? Ils restent avec leurs épouses non juives, engendrent des fils dont la condition est la même que celle de leur mère, pour être assimilés aux non-juifs. J'ai préparé une résolution et l'ai envoyée avec l'opinion du rabbin Aharon Halevi Goldman au rabbin Joseph Yadid Halevi, président du tribunal rabbinique de la communauté d'Alep à Jérusalem. Ce dernier ratifia notre rapport. J'ai dès lors diffusé l'annonce selon laquelle toute conversion devait être pour toujours interdite en Argentine, pour les raisons évoquées par nous trois. Celui qui voudrait se convertir pourra aller à Jérusalem. Peut-être là-bas le tribunal acceptera le candidat ${ }^{22}$. 
«La logique sous-jacente de cette interdiction - écrit Daniel Bargman - repose sur l'idée qu'il manquait dans le nouveau cadre de vie argentin un contexte communautaire donnant sens et légitimité aux conversions. Cela impliquait la rapide reconnaissance du caractère séculier de la majeure partie de la communauté juive locale, faisant de l'entrée dans la vie religieuse un acte hypocrite aux yeux des autorités rabbiniques. Les conceptions de la sainteté et l'exclusion visent la démarcation - de la part de ceux qui fixent les normes du groupe - de frontières rigides, refusant l'entrée de nouveaux membres et excluant ceux qui avaient transgressé la prescription endogamique. "Étant donné leur infraction - sanctionnent les rabbins - ils se mettent eux-mêmes en dehors de la communauté". En conséquence, ils appellent à "affermir le cercle de sainteté vis-à-vis de l'intérieur et de l'extérieur, pour maintenir les déviants éloignés des saintes institutions". ${ }^{23}$

Selon cette interprétation explicitement inspirée des théories de Fredrik Barth ${ }^{24}$, l'interdiction formelle de célébrer des conversions sur le sol argentin aurait comme vocation première d'affermir les frontières du groupe face au danger que représente l'assimilation. L'exclusion des déviants renforcerait ainsi la cohésion interne du groupe selon une logique proprement sectaire où chaque membre est - idéalement du moins, et pour reprendre une terminologie wébérienne - un « virtuose ». Le rappel inconditionnel de la règle d'endogamie viserait à privilégier la qualité de l'engagement au détriment de la quantité de fidèles, l'important étant, en dernière instance, qu'un noyau, aussi infime et minoritaire soit-il, pérennise l'engagement entier et sincère envers les mitsvot.

Certains éléments poussent cependant à enrichir cette interprétation. En 1915, soit douze ans avant que soit prononcée l'interdiction totale des conversions, le rabbin Setton était allé chercher les conseils de son homologue ashkénaze Aharon Halevi Goldman (cosignataire du décret de prohibition) en charge de la communauté de Moisesville à propos du cas d'un jeune homme syrien qui, sans autre motif apparent, voulait se convertir auprès du bet din de la communauté Hesed Schel Emeth Sefaradit de Buenos Aires que dirigeait le rabbin Setton.

Aharon Halevi Goldman, émit la réponse suivante :

Selon moi et selon toute personne probe, la calamité s'est propagée ici ; la plupart de nos frères ont abandonné la Torah et les rigoureuses mitsvot se sont transformées en choses superficielles. Ces transgresseurs sont si nombreux que s'il se trouvait un juif pour observer le Shabbat et les autres mitsvot il pourrait être considéré comme un Tsadik [un juste]. Ils sont si peu nombreux qu'un enfant pourrait en faire la liste. Maintenant imaginez, si nous prévenons le converti de tout ce qu'engagent les mitsvot et que plus tard il observe comment la plupart d'entre nos frères les transgressent, il se demandera sûrement : que signifiaient tous ces avertissements des rabbins d'Israël ? La maison d'Israël n'est-elle pas justement comme celle des gentils?

En conséquence, nous nous montrons irresponsables quand le converti avilit les préceptes du judaïsme et, également, quand une personne s'unit à nos ennemis pour être « des aiguilles devant vos yeux et des épines à vos côtés »... Pour cela, je ne veux rien avoir à faire avec ce cas ${ }^{25}$.

17 Le raisonnement du rabbin repose sur la conviction profonde qu'aucune conversion ne devrait être effectuée quand les juifs eux-mêmes se tiennent éloignés de la Torah : dans cette réponse, il ne fait à aucun moment référence aux mariages mixtes, et c'est bien l'inobservance religieuse qui est dénoncée en premier lieu par ce commentaire. Selon la même logique, le décret de prohibition prononcé douze ans plus tard par le rabbin Shaul 
David Setton non seulement fustige l'inobservance religieuse des juifs argentins (sa lettre commence en effet par souligner que «La vie à Buenos Aires est particulièrement débauchée et [que] chacun y fait ce qui lui plait»), mais également déplore l'absence d'autorité capable d'imposer une norme religieuse commune à l'ensemble des congrégations juives du pays («[...] il n'y a aucune autorité rabbinique écoutée et respectée, ni de rabbin nommé par le gouvernement ou par la communauté juive ellemême " poursuit-il). Il semble donc bien que le décret vise autant, sinon plus, à structurer un champ religieux juif argentin anomique (en terme d'institutions représentatives et de comportements individuels) qu'à lutter efficacement contre les mariages mixtes en imperméabilisant les frontières. L'enjeu est bien d'imposer - ou au moins d'énoncer - une norme forte à l'ensemble des communautés argentines, que ce soit en terme de logiques matrimoniales (et plus largement de définition de l'identité), que de normes religieuses. Le décret s'inscrit dans une logique de réaffirmation, à l'ensemble des congrégations argentines, de règles perçues comme fondatrices de l'identité juive. Il a une portée plus universelle que sectaire : il réaffirme la centralité de la loi pour l'ensemble des juifs argentins désirant vivre collectivement leur identité.

La lente réception du décret (1930-1960)

L'adoption du décret par l'ensemble des congrégations ne fut pas immédiate. La soumission au décret impliquait, pour les tribunaux rabbiniques, une relative perte d'autonomie, condition qui avait jusque-là fait obstacle à toutes les tentatives de fédération institutionnelle du champ religieux juif argentin ${ }^{26}$. Elle ne se fit donc que progressivement, sous l'effet de rapports de force intra et inter communautaires, ainsi que par l'intervention de prestigieuses autorités rabbiniques étrangères.

Suivons le cas de l'adoption du décret par la Comunidad Israelita Sefaradí. Cette communauté regroupant les juifs originaires de Turquie sollicita en 1930 la communauté Hesed Schel Emeth Sefaradit pour qu'elle lui vende une partie de son cimetière. Les juifs alépins exigèrent en retour que les Turcs soumettent à leur tribunal rabbinique l'ensemble des cas de mariages mixtes de la Comunidad Israelita Sefaradí afin qu'il juge de leur validité halakhique. Il s'agissait de statuer sur toutes ces « conversions » prononcées bien des années avant la prohibition du rabbin Setton ${ }^{27}$. Ils exigeaient également que la Comunidad Israelita Sefaradí se soumette au décret du rabbin Setton et bloque toute conversion, ce en quoi les rabbins se montraient réticents. Ces derniers réalisèrent ainsi durant la première moitié de la décennie 1930 des conversions ainsi que des mariages dont l'un des conjoints avait été converti. Céder aux exigences de la communauté voisine signifiait pour les rabbins de la Comunidad Israelita Sefaradí reconnaître une moindre compétence à leur tribunal rabbinique. Affirmer l'incompétence de l'ensemble des bet din argentins en matière de conversion, c'était en effet les disqualifier partiellement en les cantonnant dans des registres de moindre portée juridique. En cela, le pouvoir de convertir apparait bien comme la plus haute fonction des dayanim - des juges - mais également comme leur distinction ultime : un bet din qui n'a pas ce pouvoir est un bet din qui perd une large part - ou peut-être même la plus large part - de son autorité.

Les rabbins de la Comunidad Israelita Sefaradí hésitaient donc à se priver d'une de leurs principales fonctions de pouvoir et de prestige. Et de manière particulièrement intéressante, c'est sous la pression de leurs propres sociétaires qu'ils durent rendre les armes et fermer définitivement la porte des conversions. En janvier de l'année 1936, 67 d'entre eux présentèrent une pétition stipulant : 
Les soussignés, considérant l'extraordinaire importance que revêt quotidiennement entre les membres de la communauté le problème de l'assimilation, et considérant que même sans entrer dans des considérations religieuses, l'interdiction et la stricte limitation des conversions peuvent reposer sur des fondements séculiers comme : $1^{\circ}$ - les nombreux doutes qui surgissent immédiatement sur la fermeté des convictions de la part de personnes qui abjurent les croyances que leurs parents leur ont inculquées, et $2^{\circ}$ - la recrudescence, à l'heure actuelle, des persécutions antisémites qui nous pousse à maintenir fermement unie la communauté, sans aucune désertion d'aucun type; nous proposons: $1^{\circ}$ - Que dès à présent soit interdit au premier officiant ou à quelconque autre employé de l'institution de pratiquer des conversions ou de bénir des mariages de convertis, sous peine de renvoi ; $2^{\circ}$ - et qu'en conséquence, tout membre qui célèbrera des conversions ou consentira à un mariage avec une personne convertie ou appartenant à une autre religion, sera déclaré par la société déshonoré et sera passible des mesures extrêmes prévues par l'article 12 de nos statuts (l'exclusion). Le chef de famille sera déclaré consentant... ${ }^{28}$

21 L'adoption d'une politique de fermeture absolue en matière de mariages mixtes et de conversion fut défendue et obtenue non pas par les autorités religieuses, mais par des «laïcs » soucieux de préserver l'intégrité «ethnique» de la communauté. Les motifs justifiant l'exclusion de tout contrevenant à la règle d'exogamie s'appuient en premier lieu sur des arguments très éloignés de la Halakha - sans doute aussi parce, étant précisément des laïcs, leur argumentaire ne pouvait se fonder sur la halakha - : le fait même de se convertir implique une présomption d'infidélité (puisqu'ils abjurent la religion de leurs parents, les convertis sont marqués du sceau de l'inconstance et doivent être légitimement suspectés de ne pas être sincères...); et la recrudescence de l'antisémitisme nécessite plus que jamais de souder la communauté autour de liens solides. En définitive, la diffusion du décret de prohibition nécessitait de renverser la tendance "congrégationaliste» du judaïsme argentin, ce qui impliquait l'émergence d'une autorité capable de transcender les pouvoirs locaux.

Dans le cas de la Comunidad Israelita Sefaradí, cela put se faire par la convergence de deux logiques en apparence contradictoires : celle des rédacteurs du décret visant à affirmer la sainteté du peuple d'Israël uni autour d'une seule autorité commune : le texte sacré et les mitsvot; et celle des membres de la congrégation entendant affirmer l'homogénéité « ethnique » de la communauté (affirmation que permettait sans peine le décret). La diffusion du décret à l'ensemble du champ religieux juif argentin ne fut cependant achevée que par le renfort d'autorités religieuses extérieures. En 1937, au retour d'un voyage en Argentine, le rabbin Hiskia Shabetay (qui avait dirigé la communauté syrienne d'Alep durant 18 ans et qui était à l'époque l'un des principaux rabbins séfarades de Jérusalem) examina, avec le soutien du grand rabbin de Palestine Isaac Herzog et du grand rabbin de Jérusalem Tzvi Pesah Franck, la validité du décret. La question la plus épineuse concernait son éventuelle rétroactivité : la prohibition devait-elle concerner toutes les conversions réalisées depuis l'arrivée des juifs en Argentine ou ne prendre effet qu'à partir du moment de son énoncé ?

23 Après de longues discussions, ils plaidèrent pour le maintien de la prohibition et pour sa rétroactivité. Mais cela ne pouvait coïncider qu'avec une réévaluation a posteriori des cas de conversion antérieurs et, afin d'éviter à ces nombreuses familles un long et coûteux voyage en Israël, ils proposèrent de « délocaliser » le bet din de Jérusalem à Buenos Aires afin de procéder - après un examen au cas par cas - à des cérémonies de « reconversion " ${ }^{29}$. Par ailleurs, en 1938, le rabbin Shabetay recommandait à travers une communication à 
l'intention de toutes les communautés argentines que soient exclus du décret les enfants nés de ces unions halakhiquement douteuses et qu'ils soient accueillis à bras ouverts dans les écoles et les communautés ${ }^{30}$. Le renfort de légitimité que procurait l'aval d'autorités aussi prestigieuses que les grands rabbins de Palestine et de Jérusalem incita les autorités de la communauté alépine de Buenos Aires à relancer l'offensive afin de convaincre les autres rabbins argentins. Dès réception de l'avis du rabbin Shabetay, ils distribuèrent un feuillet rappelant ces dernières décisions juridiques.

Fort de cette légitimité, le décret fut progressivement respecté officiellement par l'ensemble des rabbins du pays, à commencer par le rabbinat central de la AMIA fondé en 1940 et représentant jusque dans les années 1960 la grande majorité des synagogues argentines. Pensé au départ comme une réponse à la prolifération des mariages mixtes et des conversions "sauvages", ce cas inédit d'interdiction des conversions permit bien davantage de normer le judaïsme argentin et de l'aligner sur des pratiques officielles orthodoxes. Il fut un instrument fondamental d'une homogénéisation religieuse qui, si elle n'était pas entièrement réalisée au niveau institutionnel, était devenue réelle au niveau idéologique. Cette "orthodoxisation » de la vie religieuse argentine fut rendue possible grâce à la convergence de deux logiques distinctes: la logique religieuse des promoteurs du projet, et la logique ethnique de certaines communautés, séfarades notamment. Mais elle ne put s'imposer que par l'intervention d'autorités extérieures émanant de centres prestigieux du judaïsme, seules à même de dépasser les rivalités locales.

Ce rappel du respect absolu de la loi tira ainsi la norme religieuse vers plus d'orthodoxie et brisa la logique congrégationaliste sur laquelle s'était, dans les premiers temps, construit le judaïsme argentin. En ce sens, le «levier» de la prohibition agit comme un outil au service de ce que l'on pourrait appeler une homogénéisation idéologique - à défaut d'être une centralisation institutionnelle - du champ religieux juif argentin. Elle permit de le regrouper sous le dais d'un référent commun même si, dans les faits, les pratiques variaient sensiblement selon les communautés et congrégations.

La pluralisation idéologique du judaïsme argentin (de 1960 à nos jours)

L'homogénéité idéologique du judaïsme argentin ne résista pas longtemps à la dynamique de pluralisation qui touchait l'ensemble des grandes diasporas juives mondiales. L'absence de réelle unité institutionnelle la rendait fragile et vulnérable aux tentatives de pénétration des courants non orthodoxes venus notamment du nord du continent américain. Malgré les efforts en grande partie productifs de certains rabbins du pays pour imposer les critères de l'orthodoxie à l'ensemble des communautés, l'engagement des juifs argentins dans les dimensions religieuses de leur judéité restait globalement faible et plus traditionaliste que véritablement orthodoxe. Si les autorités religieuses étaient donc plus ou moins orthodoxes, les fidèles, de leur côté, entretenaient un rapport souvent lâche, festif et symbolique avec la tradition, sans toutefois que ce rapport soit réellement théorisé.

La Congregación Israelita de la República Argentina (CIRA) - la plus ancienne institution du pays - incarnait parfaitement ce rapport au religieux. Ses premiers temps avaient été marqués, tant du côté de l'institution que de celui des fidèles, par une relative mise à distance des principes halakhiques, qui donnait lieu à de nombreux arrangements comme la multiplication des conversions «sauvages ». En somme, cette institution fonctionnait typiquement sur le modèle congrégationaliste que nous mentionnions en début d'article : le rabbin était certes le plus qualifié, mais cette qualification n'excédait pas quelques 
principes de base. La cohérence religieuse et rituelle ne pouvait dans ce cas s'opérer que par la mise en commun des différentes compétences réunies dans la synagogue, diffusant l'autorité à l'ensemble de la communauté.

Dès 1910 cependant, les membres de la CIRA se préoccupèrent de revitaliser leurs pratiques religieuses et envoyèrent leur rabbin (à l'époque Samuel Halphon) suivre un cycle de formation auprès du séminaire rabbinique du Consistoire central de France ${ }^{31}$. Cela représentait un premier pas vers l'orthodoxisation de la congrégation, mais signifiait aussi l'adoption $d u$ modèle consistorial $d u$ franco-judaïsme qui reposait, dans sa formulation historique, sur l'idée d'une certaine tolérance vis-à-vis de la déviance religieuse ou, plus précisément, de la pluralité des pratiques. En tant qu'institution « universelle » ayant vocation à représenter l'ensemble des juifs de France, le Consistoire de France accueillait en son sein une multitude de sensibilités et permettait ainsi la coexistence de différentes modalités d'investissement religieux, allant du plus orthodoxe au plus relâché. En définitive, il s'agissait bien d'une institution de type Église, tant dans son organisation (hautement centralisée) que dans sa vocation ${ }^{32}$. La religiosité institutionnalisée par la CIRA à partir des années 1910 s'inscrivait bien dans une religiosité que l'on peut qualifier de "traditionnelle " ${ }^{33}$, marquée à la fois par une certaine distance vis-à-vis des prescriptions et par une volonté affichée de rester dans les cadres légitimes de la tradition (ce qui impliquait par exemple le respect de la prohibition des conversions).

L'année 1947 bouleversa cette " tranquillité » traditionnelle dans laquelle vivait la CIRA. Les responsables communautaires, inquiets de constater la désertion croissante des jeunes, se préoccupèrent à cette époque de développer de nouvelles activités communautaires à leur intention et chargèrent Adolpho Weil (qui devint plus tard président du Seminario Rabínico Latinoamericano et publia en 1988 une histoire du judaïsme conservateur en Argentine ${ }^{34}$ ) de superviser l'opération. Celui-ci s'adressa en premier lieu au Jewish Theological Seminary de New York (conservative) afin de s'inspirer de ses méthodes. Il créa ainsi dès 1955 un groupe appelé Círculo Cultural y Social (Cercle culturel et social) à destination des jeunes couples, un groupe de femmes, des cours gratuits de préparation à la bar mitsva, un jardin d'enfants et une bibliothèque ${ }^{35}$. Dans le même mouvement, des voix (féminines) s'élevèrent pour initier des cérémonies de bat mitsva (pour les jeunes filles).

30 Selon les souvenirs d'A. Weil, ces initiatives connurent un succès rapide mais ne permirent cependant pas de diriger ces jeunes vers les offices shabbatiques, qu'ils trouvaient ennuyeux et désuets. Au mois de janvier 1956, la Congregación reçut la visite surprise - du rabbin conservative Morris Silverman, de New York, qui, de passage à Buenos Aires pour ses vacances, fut accueilli à bras ouverts et invité aux offices de fin de semaine. Il incita alors la CIRA à adhérer au mouvement conservateur. Il proposa également de faire traduire en espagnol un Siddour ${ }^{36}$ (évidemment conservateur) afin que tous les membres de la congrégation puissent se référer au même texte.

31 La proposition fut cependant rejetée par l'assemblée communautaire - et notamment par la vieille garde - qui n'entendait pas rompre officiellement avec l'orthodoxie en s'affiliant formellement avec un courant désigné par les institutions orthodoxes comme hétérodoxe. Une année plus tard, la CIRA vit arriver un autre rabbin du Jewish Theological Seminary de New York, qui passait également à Buenos Aires pour des vacances. Adolpho Weil raconte : «Il se passa une année et, durant le mois de janvier 1957, la congrégation reçut lors du cabalat shabbat la visite surprise de Maxwell Abbel, président de la United 
Synagogues of America [institution centrale du judaïsme conservative], accompagné de son épouse, qui effectuaient un voyage d'agrément en Amérique du Sud sur les conseils du rabbin Silverman. [...] Maxwell Abbell se montra très bien informé par le rabbin Silverman de la situation du judaïsme dans cette partie du continent. De longues conversations autour du problème de l'adhésion de notre institution au mouvement conservateur nous révélèrent un homme intelligent et conscient de la tendance décadente et assimilationniste de la majeure partie des nouvelles générations juives en Amérique du Sud si n'étaient pas urgemment prises les décisions adéquates et effectives pour la contrecarrer. ${ }^{37}$

Ce séjour fut l'occasion pour le Jewish Theological Seminary de renouveler et d'enrichir son offre de collaboration en proposant d'envoyer sur place un jeune rabbin. Marshall Meyer arriva à Buenos Aires en août 1959. Son dynamisme et surtout son charisme provoquèrent une véritable émulation au sein de l'antique CIRA. Non seulement les jeunes participaient activement aux activités qui leur étaient spécialement dédiées, mais ils affluaient de plus en plus nombreux aux offices shabbatiques. "Un vent nouveau» soufflait sur la communauté - écrit Adolpho Weil ${ }^{38}$ - ; le jeune rabbin apportait non seulement une compétence réelle - professionnelle - en matière d'activités à destination de la jeunesse, mais tentait également d'imposer certaines pratiques issues directement de la formulation idéologique conservative, comme la mixité des offices organisés spécialement pour les jeunes. Ces innovations étaient accueillies fraîchement par toute une frange de la congrégation. Le rabbin principal Schlesinger, convaincu par certains aspects de l'efficacité de la démarche, mais plus réticent pour certains autres - et aussi contraint de ménager la partie la plus traditionaliste de sa communauté - rappela à l'ordre le fougueux rabbin. Ce dernier n'en continua pas moins son travail, qui s'étendait dans deux directions principales: la conquête de la jeunesse et la consolidation de l'assise conservative sur le sol argentin.

Le 14 août 1960 - soit presque un an jour pour jour après l'arrivée de Marshall Meyer à Buenos Aires -, une convention regroupant différents rabbins argentins résolut de fonder le Seminario Rabínico Latinoamericano, l'objectif étant de créer une institution capable de former des rabbins hispanophones compétents et... conservateurs. Il ouvrit sa première classe en avril 1962 dans les locaux de la CIRA, avec deux étudiants ashkénazes et deux étudiants séfarades. L'affiliation progressive de la Congregación Israelita de la República Argentina au courant conservateur permettait ainsi son décloisonnement «ethnique ». Jusqu'à cette époque, elle regroupait essentiellement la judaïcité ouest-européenne (alsacienne et allemande) ; elle ouvrait désormais ses portes à d'autres origines nationales et rituelles et annonçait une tendance progressive à l'idéologisation du judaïsme argentin et, plus largement, sud-américain - c'est-à-dire à sa structuration progressive en institutions séparées et concurrentes dont les lignes de fractures ne reposent plus sur des différences rituelles, nationales ou culturelles, mais sur des définitions différentes de la vérité religieuse, autrement dit des conceptions différentes de l'« orthodoxie ».

Parallèlement à la fondation du Seminario Rabínico Latinoamericano, les offices de Shabbat destinés spécifiquement aux jeunes ne désemplissaient pas et commençaient à attirer un grand nombre d'adultes ${ }^{39}$, si bien qu'ils se posaient en véritables concurrents des offices traditionnels. La tension devint sensible. En 1963, pour la première fois dans l'histoire de la congrégation, deux listes furent déposées pour l'élection partielle de la commission directrice. Plutôt que d'entrer en guerre ouverte au sein même de la CIRA, ceux qui composaient la liste favorable au changement retirèrent leur candidature, mais se mirent 
d'accord pour fonder leur propre institution synagogale, qu'ils nommèrent Comunidad Bet $\mathrm{El}$, et qui fut officiellement affiliée au mouvement conservative mondial. Le rabbin Marshall Meyer, qui avait posé sa démission deux mois auparavant, se laissa convaincre de devenir le rabbin de la nouvelle communauté. Celle-ci prit en charge la gestion du Seminario Rabínico Latinoamericano, qui fut doté dès le 11 septembre 1969 d'un centre autonome au 1760 de la rue José Hernández, dans le quartier cossu de Belgrano. Le Seminario Rabínico Latinoamericano - qui représente le judaïsme conservateur pour l'ensemble de l'Amérique latine - regroupe aujourd'hui environ 80 communautés, dont la majorité en Argentine, et a su fidéliser de nombreux juifs qui jusque-là restaient éloignés des dimensions religieuses de leur identité. Son succès fut donc rapide, au point d'apparaître aujourd'hui comme l'une des institutions dominantes au sein du champ religieux juif argentin.

On retiendra d'abord de l'histoire de cette implantation le rôle central joué par le très charismatique rabbin Marshall Meyer. C'est bien en effet selon une dynamique proprement charismatique qu'a pu être fondée cette institution: partant d'une stratégie d'entrisme consistant à "convertir» une structure déjà existante à l'idéologie conservative, ce jeune rabbin s'est - dans un schéma très wébérien - rapidement posé en force concurrente du pouvoir traditionnel - qui en retour s'est alors constitué en défenseur de l'orthodoxie - jusqu'à être en mesure de fédérer un groupe suffisamment important pour provoquer une scission. L'affirmation de la présence du judaïsme conservateur dans le champ religieux juif argentin impliquait par ailleurs la remise en cause explicite du décret de prohibition. Ne pas le remettre en question, maintenir fermée la porte des conversions, c'était se priver de toute autorité juridique. De plus, l'affiliation au mouvement conservateur signifiait l'adhésion à ses valeurs, qui comprennent, entre autres, la mixité des offices, l'accès des femmes au rabbinat et le recours aux conversions, notamment dans le cadre de mariages mixtes ${ }^{40}$. Dans cette logique, le Seminario Rabínico Latinoamericano entreprit rapidement d'ouvrir un mikvé et de convertir ses premiers candidats, violant ouvertement le décret du rabbin Setton.

Un champ pluriel et compétitif

La naissance du Seminario Rabínico Latinoamericano initia un mouvement plus général de pluralisation idéologique et institutionnelle du champ religieux juif argentin. Deux ans après la fondation de la communauté Bet $\mathrm{El}$, et à la suite d'une scission avec cette dernière, une seconde communauté moderniste ouvrit sous le nom de Emanu-El, qui resta toutefois affiliée au Seminario Rabínico Latinoamericano. L'une des conséquences majeures de ce mouvement de pluralisation idéologique et institutionnelle fut de faire de l'orthodoxie - et de ses principales institutions comme par exemple le Grand Rabbinat orthodoxe ashkénaze de la AMIA - une dénomination parmi d'autres devant désormais faire face à une concurrence et une compétition croissantes.

À partir du début des années 1970, le vif succès que rencontrait notamment la communauté Bet $\mathrm{El}$ (qui fédéra rapidement plus de 1000 familles) ainsi que les autres communautés affiliées au Seminario Rabínico Latinoamericano polarisèrent la compétition idéologique au centre. En proposant de composer avec certaines valeurs de la modernité tout en tâchant de maintenir une certaine exigence religieuse, le mouvement conservative rencontrait le désir d'une part non négligeable de la population juive de Buenos Aires, qui pouvait envisager son affiliation conservative comme une perspective crédible. Par ailleurs, la possibilité offerte par cette institution aux couples mixtes d'homogénéiser religieusement leur couple par la conversion concurrençait directement les institutions 
orthodoxes qui, à travers l'application stricte du décret de prohibition, excluaient de leurs communautés un nombre significatif de familles dont le couple parental, sans être nécessairement religieusement homogène, n'en désirait pas moins transmettre l'identité juive à ses enfants ${ }^{41}$.

La pluralisation des offres religieuses juives nécessitait ainsi d'introduire davantage de souplesse dans l'application du décret du rabbin Setton. En 1966, le rabbin David Kahana prit la direction du bet din de la AMIA (orthodoxe) et entreprit des démarches auprès de sommités halakhiques israéliennes afin d'étudier les possibilités d'annulation du décret ${ }^{42}$. Une solution fut trouvée par le rabbin Joshua Menachem Ehrenberg de Tel Aviv. Considérant qu'en l'état actuel du droit, ceux qui voulaient se convertir devaient se présenter devant le tribunal rabbinique israélien, la prohibition pouvait être contournée en envoyant des émissaires du bet din de la Rabanout israélienne valider sur place les conversions (solution qui avait d'ailleurs été envisagée en 1937 par le rabbin Hiskia Shabetay - voir supra -). Il suggéra même que ces démarches pouvaient être réalisées par correspondance, le rabbinat argentin envoyant une requête auprès de la Rabanout et celle-ci faisant simplement connaître sa décision par retour du courrier ${ }^{43}$. L'idée était de mettre sous tutelle le bet din argentin, ce qui permettait de sauver formellement la décision du rabbin Setton et de contrôler le flux des conversions. Il ne s'agissait pas en effet de rompre purement et simplement l'interdit, mais de permettre à un tribunal rabbinique argentin - et non à tous les tribunaux rabbiniques argentins - de le contourner, sous le contrôle de la haute autorité israélienne. Selon Moshe Zemer, un certain nombre de conversions par correspondance furent ainsi réalisées, dont les bénéficiaires furent reconnus comme juifs pas l'ensemble des tribunaux rabbiniques orthodoxes ${ }^{44}$.

Encore faut-il être prudent. La conversion, en Argentine comme dans l'ensemble du judaïsme, est un domaine à la fois tabou et polémique. Moshe Zemer est par exemple membre du bet din de l'Israel Council of Progressive Rabbis (de tendance libérale) et a ainsi tout intérêt à démontrer la caducité du décret de prohibition en affirmant que même les rabbins orthodoxes le contournent. Les institutions orthodoxes ont de leur côté tout intérêt à s'en tenir à une position officielle de respect du décret (qui leur permet d'accuser les institutions concurrentes d'hétérodoxie et de laxisme, et ainsi de se poser comme les garants responsables de la tradition) tout en facilitant officieusement la conversion des candidats qu'ils jugent dignes.

En somme, la politique en matière de conversion est instrumentalisée d'un côté comme de l'autre pour contester ou consolider le monopole. Pour le Seminario Rabínico Latinoamericano, le fait de proposer des conversions aux couples rejetés par les autres institutions lui permet de grossir les rangs de ses offices et ce faisant de se poser comme une institution alternative et crédible. Par ailleurs, le gain démographique que cela lui procure lui donne un poids supplémentaire en termes de légitimité nationale. Enfin, l'attitude compréhensive dont elle fait preuve à l'égard des couples mixtes rejetés par les rabbins orthodoxes lui donne un "supplément d'âme» en affinité avec les traits de la modernité culturelle. Du côté des institutions orthodoxes, il s'agit de tenter de monopoliser le droit de convertir en maintenant officiellement la validité de la prohibition tout en s'attribuant le droit exclusif de la contourner. En somme, elle s'aligne sur la politique de son concurrent (en renouant de fait avec une politique d'ouverture envers les candidats à la conversion) tout en s'efforçant de lui couper l'herbe sous le pied en lui déniant le droit de mettre en pratique des principes qu'il a lui-même impulsés. 
Cette politique a d'ailleurs un succès certain, dans la mesure où l'orthodoxie garde en Argentine la main sur certains biens symboliques de valeur. Les quatre plus importants cimetières sont gérés par des institutions de sensibilité orthodoxe, qui interdisent l'accès aux convertis «argentins " - à l'exception de ceux validés par le bet din israélien. Et, encore maintenant, ces convertis non orthodoxes ne sont pas reconnus par la Rabanout israélienne, ce qui leur interdit notamment tout mariage sur le sol israélien ${ }^{45}$.

En conclusion

41 Le décret de prohibition des conversions du rabbin Shaul David Setton n'eut qu'une incidence marginale sur le nombre de mariages mixtes qui, aujourd'hui encore, s'approche des $40 \%$. En revanche, comme l'épopée de ce décret nous l'a montré - depuis sa promulgation, son adoption et sa contestation -, il devint rapidement le point à partir duquel allait s'organiser le champ religieux juif argentin. Il est particulièrement révélateur de la place paradoxale qu'occupe la question des conversions dans le judaïsme contemporain. Les mariages mixtes, l'inobservance religieuse, l'affirmation de l'autonomie individuelle et la pluralisation des pratiques et des vérités données à croire, constituent les principaux enjeux auxquels tentent de répondre les différentes autorités religieuses contemporaines. Autant de dimensions que cristallise la question des conversions dans la mesure où définir les modalités d'acceptation de ceux qui frappent à la porte du judaïsme, c'est tracer une frontière entre juifs et non juifs bien sûr, mais c'est aussi proposer une définition normative de l'identité juive (les rabbins, convertissent ceux qu'ils jugent conformes à l'idée qu'ils se font du «bon juif») et un niveau de conformité minimale aux prescriptions rabbiniques. Mais plus encore, dans le cadre de sociétés largement sécularisées, le fait de pouvoir ou non accorder la conversion constitue le dernier pouvoir dont disposent les autorités religieuses. Convertir, pour ces institutions religieuses, c'est par excellence exercer l'autorité. À travers le processus de conversion peut s'expliciter un pouvoir qui, sous l'effet de la sécularisation, se réduit à une peau de chagrin.

Dans cette logique, un tribunal rabbinique dont les conversions ne sont pas reconnues par les autres tribunaux est un tribunal de moindre autorité, tant à l'intérieur de sa communauté que vis-à-vis des autres tribunaux rabbiniques. Il existe donc un « pouvoir de convertir ", non pas seulement en direction des candidats à la conversion, mais dans le cadre d'une pluralisation croissante du judaïsme en courants formulant des « orthodoxies » concurrentes. De la légitimité des conversions prononcées par un tribunal rabbinique dépend ainsi plus largement sa légitimité comme tribunal rabbinique compétent et souverain. C'est bien là que se situe l'enjeu du décret de prohibition ainsi que de sa contestation: définir le légitime et l'illégitime au sein du judaïsme argentin. Mais il prend dans ce cas précis un caractère paradoxal : en privant de toute compétence en matière de conversion l'ensemble des tribunaux rabbiniques argentins, le décret en a fait des tribunaux vassalisés aux tribunaux israéliens, sans réel pouvoir et sans réelle autorité. La contestation du décret elle-même par le Seminario Rabínico Latinoamericano, loin de s'affirmer comme un acte d'autonomisation et de souveraineté, a pris la forme d'une allégeance au judaïsme conservative d'origine nord-américaine. Judaïsme du bout du monde, le judaïsme argentin apparaît ainsi, malgré son poids démographique certain, comme un judaïsme sous tutelle, un judaïsme périphérique. Et pour une grande part, les oppositions idéologiques qui le traversent aujourd'hui et qui s'expriment à travers la conversion sont la transcription presque parfaite de celles qui opposent de manière de plus en plus affirmée les deux grands centres du judaïsme contemporain: Israël d'une 
part, dominé par les institutions orthodoxes, et les États-Unis d'autre part, où la grande majorité des juifs sont membres des synagogues modernistes.

\section{NOTES}

1. Daniel ElaZAR, Jewish Communities in Frontier Societies. Argentina, Australia, and South Africa, New York, Holmes and Meyer, 1983, p. 89-90.

2. Victor A. Mirelman, En busqueda de una identidad. Los inmigrantes judíos en Buenos Aires 1890-1930, Buenos Aires, Milá, 1988, p. 3.

3. C'est ainsi qu'est souvent dénommée la ville de Buenos Aires.

4. Ricardo Feierstein, Historia de los judíos argentinos, Buenos Aires, Ameghino, 1993, p. 109.

5. Victor A. Mirelman, op. cit., p. 6.

6. Voir Haim Avni, Argentina y la historia de la inmigración judía (1810-1950), Buenos Aires, Magnes, 1983, p. 135-142 et p. 208-237.

7. Haim AvNi, ibid., p. 401-479.

8. Ibid. On compte aussi environ un millier de juifs italiens (voir Eleonora Maria Smolenski et Vera Vigevani Jarach, Tantas voces, una historia. Italianos judios en la Argentina 1938-1948, Buenos Aires, Temas, 1999).

9. Doris Bensimon, Les juifs dans le monde au tournant du XXI siècle, Paris, Albin Michel, (coll. « Présence du judaïsme poche », 1994, p. 56.

10. Ce chiffre m'a été donné par certains chercheurs argentins spécialisés sur la question et par les dirigeants communautaires rencontrés lors de mes deux séjours de terrain à Buenos Aires.

11. Ricardo FEIERSTEIN, op. cit., 1999, p. 229.

12. Victor A. MiRelman, op. cit., 1988, p. 161.

13. Ibid., p. 165.

14. Ibid., p. 161.

15. Bain rituel.

16. Maïmonide écrit ainsi : « Si un converti, dont on n'aurait pas vérifié la motivation, a été circoncis et a fait l'immersion du bain rituel, quand bien même nous saurions que c'est dans un certain intérêt [autre que celui de la conversion elle-même], il sera considéré comme ayant le statut de converti. »

17. Société funéraire juive argentine, qui devint par la suite la AMIA, principale organisation juive d'Argentine, contre l'immeuble de laquelle fut perpétré l'attentat meurtrier de juillet 1995.

18. Victor A. MiRELMAN, op. cit., p. 163.

19. On disait ainsi que les juifs arrivant à Buenos Aires laissaient leurs Tefilim sur le bateau.

20. Ibid., p. 115.

21. Moshe Zemer note à propos de ce rabbin ashkénaze qu'il avait toujours été hostile à toute forme de conversion, qu'elles soient le shem shamaim ou non [Moshe Zemer, «The 
Rabbinic Ban on Conversion in Argentina ", Judaism, ou sa version espagnole : " El edicto rabinico sobre conversión en la Republica Argentina », in Maj'shavot].

22. Rabbin Shaul Setton, cité par Victor A. Mirelman, op. cit., p. 167-168, traduit par S. Tank-Storper.

23. Daniel Bargman, « Acerca de la legitimación de la adscripción étnica: dentro, fuera y en los límites del grupo judío en Buenos Aires ", Judaica latinoamerica. Estudios Históricosociales, III, Jérusalem, Editorial Universitaria Magnes, Universidad Hebrea, 1997, traduit par S. Tank-Storper.

24. Fredrik Barth, Les groupes ethniques et leurs frontières, paru en français dans : Philippe Poutignat, Jocelyne Streiff-Fenart, Théories de l'ethnicité, Paris, PUF, 1995, p. 203-249.

25. Rabino Aharon Halevi Goldman, cité par Moshe Zemer, op. cit., p. 16-17, traduit par S. Tank-Storper.

26. À l'inverse du judaïsme français, qui s'est constitué sur la base d'une extrême centralisation religieuse opposée à un relatif éparpillement des associations à caractère culturel, le judaïsme argentin s'est institutionnalisé à partir des registres culturels et politiques de l'identité juive (AMIA et DAIA), laissant le champ religieux s'organiser sur un mode pluraliste et compétitif [voir à ce propos la chronique de Victor A. Mirelman, op. cit.].

27. Ibid.

28. Ibid.

29. Ibid., p. 170.

30. Ibid.

31. Ibid., p. 386. Je rappelle que cette communauté regroupait une majorité de juifs français - alsaciens pour la plupart - et allemands venus en Argentine avant les grandes vagues migratoires.

32. Voir à ce propos le stimulant article de Shmuel Trigano, « Le concept de communauté comme catégorie de définition du judaïsme français ", Archives européennes de sociologie, Tome XXXV, $\mathrm{n}^{\circ}$ 1, 1994.

33. Et non traditionaliste, qui suppose un rapport réflexif à la tradition. Le terme traditionnel renvoie aussi à l'idée d'immédiateté du rapport à la tradition. Cela renvoie enfin aux remarques de Margaret Mead quand elle oppose religiosité « distraite » (typique de la tradition) et religiosité « sérieuse » (typique de la modernité).

34. Adolopho Weil, Origines del judaísmo conservador en la Argentina, Buenos Aires, Ediciones Seminario Rabínico Latinoamericano, 1988.

35. Ibid., p. 59.

36. Livre de prières.

37. Adolpho WEIL, op. cit., p. 64-65.

38. Ibid., p. 81.

39. Ibid., p. 84.

40. Sur les principes fondateurs du judaïsme conservateur, se référer au livre de Rivon Kryger, La Loi juive à l'aube du XXI siècle, Paris, Messer, 1995.

41. Selon les chiffres disponibles, le bet din du Seminario Rabínico Latinoamericano a converti 1340 personnes entre 1975 et 1994 ce qui, compte tenu du faible engagement religieux de la judaïcité argentine, constitue, pour les synagogues, une « part de marché » importante [Mordejai Levin, Segunda investigacion sobre las conversiones en el Seminario Rabínico Latinoamericano, Instituto de Introduccion al Judaismo, Seminario Rabínico Latinoamericano, juin 1995, p. 7].

42. Moshe ZEMER, art. cit., p. 22. 
43. Ibid., p. 23.

44. Il mentionne des archives témoignant que le grand rabbin d'Israël Isser Yehuda Unterman avait bien désigné le rabbin Kahana comme représentant de la Rabanout en Argentine [Ibid.].

45. Le droit personnel, en Israël, est de la compétence exclusive des institutions religieuses. Deux juifs doivent ainsi se marier devant la Rabanout, de sensibilité orthodoxe, officiellement chargée par l'État de l'État civil et du statut personnel des citoyens israéliens de nationalité juive (mariage, divorce, gestion des cimetières).

\section{RÉSUMÉS}

Cet article prend pour point de départ l'analyse d'un décret rabbinique prononcé en 1927 par un rabbin argentin interdisant de manière définitive toute conversion au judaïsme sur le sol de la République argentine, pour démontrer en quoi la conversion joue, dans le judaïsme, le rôle de la régulation du pluralisme religieux. Censé au départ lutter contre les mariages mixtes et les « conversions sauvages » dans un contexte de grande porosité avec le milieu non-juif, ce décret n'eut aucune incidence réelle sur le taux d'exogamie de la judaïcité argentine (qui fut, dès les débuts, de l'ordre de 40 à $50 \%$ ). En revanche, l'imposition progressive de la prohibition des conversions permit l'homogénéisation idéologique des communautés juives argentines, jusque-là relativement atomisées. Le décret du rabbin Setton se révéla un formidable levier normatif au profit d'une orthodoxisation croissante du champ religieux juif argentin. À tel point que, aujourd'hui encore, malgré un nombre infiniment restreint de demandes de conversions adressées aux institutions argentines, le respect ou le non respect de ce décret focalise l'ensemble des débats entre les différentes tendances idéologiques et institutionnelles du judaïsme argentin, notamment entre orthodoxes et institutions de sensibilité libérales.

Starting from the analysis of a 1927 rabbinical decree definitely banning any conversion to Judaism, this paper tries to demonstrate how, within Judaism, conversion operates as a tool to regulate religious pluralism. This decree was supposed to limit the number of mixed marriages and "wild conversions" in a context of high porosity with the non-Jewish milieu. But it had little influence on the rate of outside marriages among Argentinean Jews (reaching, from the start, 40 to $50 \%$ ). On the other hand, the gradual prohibition of conversions led to a growing ideological homogeneity of the Argentinean Jewish communities which had been, until then, rather fragmented. Rabbin Setton's decree proved to be a formidable normative tool leading to the growth of orthodoxy within the Argentinean Jewish milieu. This is proven by the fact that, to this day, despite the very limited demands for conversion addressed to the Argentinean Jewish authorities, the respect or non-respect of the rabbinical decree is the focus of all the debates between the various ideological or institutional factions of Judaism in Argentina, most notably between the orthodox and liberal groups.

Este artículo toma como punto de partida el análisis de un decreto rabínico pronunciado en 1927 por un rabino argentino prohibiendo de manera definitiva toda conversión al judaísmo en el suelo de la República Argentina, para demostrar de qué manera la conversión juega, en el judaísmo, como un instrumento de regulación del pluralismo religioso. Supuestamente destinada en principio a luchar contra los matrimonios mixtos y las «conversiones anárquicas », en un 
contexto de gran porosidad con el medio no-judío, este decreto no tuvo ninguna incidencia real sobre las tasas de exogamia del judaísmo argentino (que fue, desde el principio, del orden de $40 \%$ a $50 \%$ ). En cambio, la imposición progresiva de la prohibición de las conversiones permite la homogeneización ideológica de las comunidades judías argentinas, hasta entonces relativamente atomizadas. El decreto del rabino Setton se reveló un extraordinario instrumento normativo en beneficio de una ortodoxización creciente del campo religioso judío argentino. A tal punto que, aún hoy, no obstante el número muy restringido de las demandas de conversiones dirigidas a las instituciones argentinas, el respeto o el no respeto de este decreto focaliza al conjunto de los debates entre las diferentes tendencias ideológicas e institucionales del judaísmo argentino, especialmente entre ortodoxos e instituciones de sensibilidad liberal.

INDEX

Mots-clés : Argentine, conversion, judaïsme, orthodoxisation, pluralisme religieux

\section{AUTEUR}

\section{SÉBASTIEN TANK-STORPER}

Université Paris V \& Centre d'Études Interdisciplinaires des Faits Religieux 